\title{
Experimental investigation of damage identification for continuous railway bridges
}

\author{
Deshan SHAN*, Chunyu FU, Qiao LI \\ School of Civil Engineering, Southwest Jiaotong University, Chengdu 610031, China
}

\begin{abstract}
Considering the issue of misjudgment in railway bridge damage identification, a method combining the stepby-step damage detection method with the statistical pattern recognition is proposed to detect the structural damage of a railway continuous girder bridge. The whole process of damage identification is divided into three identification substeps, namely, damage early warning, damage location, and damage extent identification. The multi-class pattern classification algorithm of C-support vector machine and the regression algorithm of $\varepsilon$-support vector machine are engaged to identify the damage location and damage extent, respectively. For verifying the proposed method, both of the proposed method and the optimization method are used to deal with the measured data obtained from a specific railway continuous girder model bridge. The results show that the proposed method can not only identify the damage location correctly, but also obtain the damage extent which is consistent with the experimental results accurately. By uncoupling finite element analysis and damage identification, normalizing the index, and seeking the separation hyper plane with maximum margin, the proposed method has more favorable advantages in generalization and anti-noise. As a result, it has the ability to identify the damage location and extent, and can be applied to the damage identification in real bridge structures.
\end{abstract}

Key words: railway bridge; damage identification; support vector machine; step by step; model test

(C) 2012 JMT. All rights reserved.

\section{Introduction}

$\mathrm{C}$ atastrophic bridge collapse accidents not only cause significant loss of property, but also have a severe social impact. Therefore, the study of bridge damage detection gets more and more attention, and becomes one of the research focuses for scholars and engineers from bridge engineering fields around the world [1-4].

The damage identification of a railway bridge structure is an interdisciplinary research topic, which refers to the knowledge of weak signal detection, signal processing, engineering mechanics, bridge engineering, artificial intelligence, stochastic processes, mathematical statistics, and so on. Scholars from various countries have committed to this field and achieved a substantial amount of research results in the last 20 years [3-5].

The damage detection algorithm based on the static testing data was frequently adopted to figure out bridge operational performances [6-10]. All this kind of studies employed optimization algorithm to detect the damage, but the misjudgment of the bridge state or the damage

Received Dec. 29, 2011; revision accepted Feb. 28, 2012

*Corresponding author. E-mail: dsshan@163.com (D.S. SHAN)

(C) 2012 JMT. All rights reserved

doi: 10.3969/j.issn.2095-087X.2012.01.001 occurrence, location, and extent would arise from the local minimum of the identification results caused by the measuring error and the disadvantage of the optimization algorithm [3].

Therefore, a method combining the step-by-step damage identification scheme [11] with the statistical pattern recognition [12] is proposed to identify the railway bridge structural damage in this paper. The railway bridge structural damage identification problem is treated as a statistical pattern recognition problem, then the damage identification of railway bridge structure is solved step by step, and different statistic pattern recognition methods are adopted for each solving step. The measured data from a specific railway continuous girder bridge model test are used to test the proposed method and the optimization method.

\section{Statistical pattern recognition for rail- way bridge damage identification}

Pattern recognition [13] is widely used to classify correctly a specific quantity from others in many disciplines. And statistical pattern recognition [11] is the classification method for pattern recognition by regarding the pattern class as a collection of random vectors for the pattern. From the viewpoint of statistical pattern 
recognition, the difference between the same classes is partly caused by the ambient noise and sensors, and partly due to the randomness of the pattern itself in the damage detection of railway bridge structures.

\subsection{Damage identification process}

There are three basic problems in the railway bridge structural damage identification: the structural damage early warning, damage location, and extent identification. For large complex structures such as railway bridges, it is extremely difficult to solve these three problems directly by the optimization algorithm, and its solutions are frequently unreasonable. In order to decrease the solution difficulty and improve the accuracy of identification results, the whole process of damage detection is divided into three identification steps corresponding to these three basic problems of damage identification, and solved step by step by introducing different pattern recognition algorithms in different steps. The damage identification procedure of a railway bridge structure is briefly shown in Fig. 1.

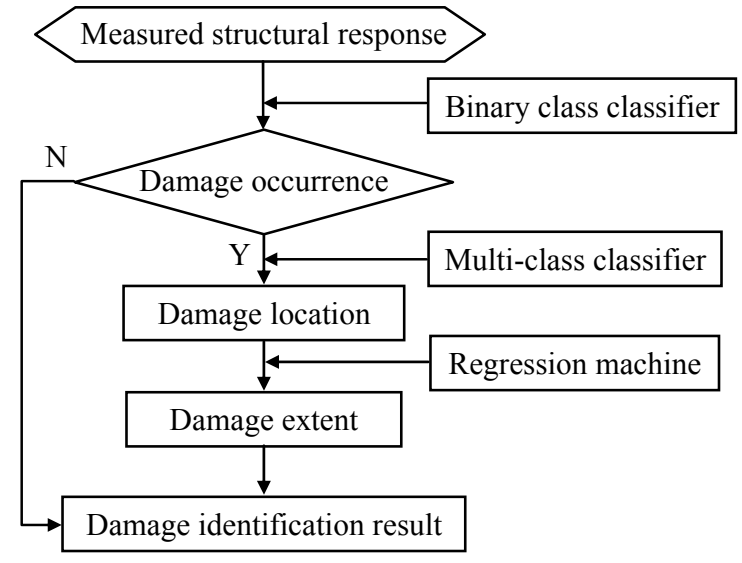

Fig. 1 Statistical pattern recognition process for damage identification of railway bridges

\subsubsection{Structural damage early warning}

Whether the railway bridge is damaged or not is a typical binary-class classification problem from the viewpoint of pattern recognition. The measured data can be obtained by the accurate instrument and loading, and these data are compared with the theoretical response of the intact bridge structure under the corresponding loading cases. If the discrepancy between the measured and theoretical responses under the same loading conditions is tolerable, and the structure is in intact state, then the identification process is terminated. Otherwise, the structure is damaged, and the damage location and extent identifications are proceeded.

\subsubsection{Structural damage location}

From the viewpoint of pattern recognition, the structural damage location identification problem is a multiclass classification problem. In light of the similarity of samples, the samples are divided into different classes by classification algorithm. As for the damage location identification, the structural response data corresponding to the same damage location is taken as the same class, and multiple classes should be figured out for different damage locations. The structural response data for different classes are obtained in advance from theoretical calculation or existing experimental results. Furthermore, multiple classes corresponding to different damage locations are formed. Then the classification model of support vector machine is established. Lastly, the model is engaged in the classification of the new measured data, and the damage location is carried out.

\subsubsection{Structural damage extent}

After locating the damage, the damage extent is identified next. The damage extent identification is a regression problem from the standpoint of pattern recognition. According to the corresponding relationship between the independent and dependent variables, the regression model is constructed by the regression algorithm, and the dependent variable corresponding to the new independent variable will be predicted by the established regression model. As for the damage extent identification, the response data are independent variables and the corresponding damage extents are dependent variables. The response data of variant damage extents for one specific damage location are pre-obtained from the theoretical calculation or existing experiment, and then the regression model of support vector machine is built up based on these datasets with variant damage extent. Furthermore, the dependent variable is predicted such that it corresponds with the new measured data by this regression model, and the damage extent is obtained.

\subsection{Damage identification implementation}

According to the pattern recognition theory, each identification step of railway bridge damage identification (early warning, damage location and extent identification) is divided into three sub-steps as well [13], namely the damage index selection, sample library construction and pattern recognition algorithm selection.

\subsubsection{Damage index selection}

The selected structural damage index should represent the structural characteristics [3]. The measured 
structural responses are generally displacements or strains in the bridge static testing, and they are related to the structural stiffness. The measured displacements or strains can be selected as the damage index. Meanwhile, damage index should be normalized for the sake of the comparability. Take $\boldsymbol{x}_{i}=\left(x_{i 1}, x_{i 2}, \cdots, x_{i n}\right)$ ( $n$ is the dimension of $\boldsymbol{x}_{i}$ ) as the damage index, and its normalization is shown as

$$
\tilde{x}_{i q}=\frac{x_{i q}}{\max _{1 \leq j \leq n}\left|x_{i j}\right|}, q=1,2, \cdots, n,
$$

where $\tilde{x}_{i q}$ is normalization result of $x_{i q}$.

\subsubsection{Sample library construction}

After the selection of damage index, the values of damage index related to different structural damaged states under possible load combination cases are calculated, and all of these values are collected in one dataset, namely the sample library.

If all the possible damage states are included in the sample library, the sample library will be tremendous in size as there are infinite damage samples in a real bridge structure. If all these infinite samples are adopted to form the sample library directly, this will result in an unreasonable amount of computing memory being required and will adversely affect the computation speed. For reducing the searching range of damage, decreasing the identification difficulty and increasing the identification accuracy, the important damage states of railway bridge structure are carried out by the structural damage sensitivity analysis. Two methods, structural mechanical properties analysis and defects investigation of railway bridge structure, are engaged in the structural damage sensitivity analysis [14]. The most probable damage states under all possible load combinations are found out through the structural mechanical properties analysis. The prone bridge defects caused by the impact of the man-made or natural environment, which could not be obtained by the structural mechanical properties analysis but certainly exist in the railway bridge structure, are obtained through the bridge defects investigation. Then, the most probable damage states and prone defects are adopted to construct the sample library to figure out the concerned damages.

The sample selection is subsequently engaged to form the sample library. The purpose of the sample selection is to maintain (or lose rarely) the generation performance of the classification algorithm, and to reduce the storage requirement and time consumption of the classification algorithm. The key samples which are helpful for classifying and predicting are selected for different classifier. For example, the decision of support vector machine is related to the support vectors, so the samples similar to the support vector are selected and the other non-support vector samples are discarded. Kernel subspace sample selection method [15] is adopted in this paper to select samples.

\subsubsection{Pattern recognition algorithm selection}

In recent decades, pattern recognition research has made a large number of achievements, and a lot of new methods have been proposed and successfully applied to many fields.

Statistical learning theory [16] is a specifically designed classifier theory for the small samples. As one of the special algorithms of statistical learning theory, support vector machine has a lot of advantages, such as optimization, the best generalization ability, etc [17]. Aided by the support vector machine algorithm, the input vector space is transformed into a new highdimensional space through the nonlinear transformation, and the optimal linear classification surface is then obtained in this new high-dimensional space.

Take $\left(\boldsymbol{x}_{i}, y_{i}\right)\left(\boldsymbol{x}_{i} \in \mathbf{R}^{n}, y_{i} \in\{-1,1\}, i=1,2, \cdots, l\right.$, where $l$ is the size of the samples) as the sample library, and the optimal linear classification surface problem can be transformed into a typical quadratic programming problem [17]:

$$
\begin{array}{ll}
\min _{\alpha} & \frac{1}{2} \boldsymbol{\alpha}^{\mathrm{T}} \boldsymbol{H} \boldsymbol{\alpha}-\boldsymbol{e}^{\mathrm{T}} \boldsymbol{\alpha}, \\
\text { s.t. } & \boldsymbol{\alpha}^{\mathrm{T}} \boldsymbol{y}=0, \\
& \boldsymbol{\alpha}^{\mathrm{T}} \geq \mathbf{0},
\end{array}
$$

where

$$
\boldsymbol{H}=\left[\begin{array}{cccc}
y_{1} y_{1} K\left(\boldsymbol{x}_{1}, \boldsymbol{x}_{1}\right) & y_{1} y_{2} K\left(\boldsymbol{x}_{1}, \boldsymbol{x}_{2}\right) & \cdots & y_{1} y_{l} K\left(\boldsymbol{x}_{1}, \boldsymbol{x}_{l}\right) \\
y_{2} y_{1} K\left(\boldsymbol{x}_{2}, \boldsymbol{x}_{1}\right) & y_{2} y_{2} K\left(\boldsymbol{x}_{2}, \boldsymbol{x}_{2}\right) & \cdots & y_{2} y_{l} K\left(\boldsymbol{x}_{2}, \boldsymbol{x}_{l}\right) \\
\vdots & \vdots & \ddots & \vdots \\
y_{l} y_{1} K\left(\boldsymbol{x}_{l}, \boldsymbol{x}_{1}\right) & y_{l} y_{2} K\left(\boldsymbol{x}_{l}, \boldsymbol{x}_{2}\right) & \cdots & y_{l} y_{l} K\left(\boldsymbol{x}_{l}, \boldsymbol{x}_{l}\right)
\end{array}\right]_{l \times l}
$$

$\boldsymbol{\alpha}=\left(\alpha_{1}, \alpha_{2}, \cdots, \alpha_{i}, \cdots, \alpha_{l}\right), \boldsymbol{\alpha}$ is the Lagrange multiplier of $\boldsymbol{x}_{i} ; \boldsymbol{y}=\left(y_{1}, y_{2}, \cdots, y_{l}\right) ; \boldsymbol{e}=(1,1, \cdots, 1) ; K\left(\boldsymbol{x}_{i}, \boldsymbol{x}_{j}\right)(i \in\{1,2, \cdots, l\}$, $j \in\{1,2, \cdots, l\})$ is the kernel function, which means the inner product of the damage index in the higher dimension space [17]. $\alpha^{*}=\left(\alpha_{1}{ }^{*}, \alpha_{2}{ }^{*}, \cdots, \alpha_{l}{ }^{*}\right)$ is the optimal solution of $\alpha$, and $\alpha^{*}$ could be obtained by solving Eq. (2). One positive component $\alpha_{m}{ }^{*}$ is selected from the all $\alpha^{*}$ components, and then

$$
b^{*}=y_{j}-\sum_{i=1}^{l} y_{i} \alpha_{i}^{*} K\left(\boldsymbol{x}_{i}, \boldsymbol{x}_{m}\right)
$$


could be calculated. As for one new input index $\boldsymbol{x}^{\prime}$, its decision making function should be expressed as

$$
f\left(\boldsymbol{x}^{\prime}\right)=\operatorname{sgn}\left(\sum_{i=1}^{l} \alpha_{i}^{*} y_{i} K\left(\boldsymbol{x}_{i}, \boldsymbol{x}^{\prime}\right)+b^{*}\right) .
$$

Although support vector machine is developed from the solving classification problem or pattern recognition problem, it can also be extended to solve the regression estimation problem. One key idea is to introduce the loss function in its extension, and then the regression problem is transformed to the classification one aided by the loss function. Therefore, the support vector machine algorithm can be used to realize the parameters regression estimation [17].

The selections of algorithm and kernel function play a vital role in the application of support vector machine. With the in-depth study of support vector machine, many transformed algorithms are proposed besides the standard support vector machine algorithm, such as Csupport vector machine, least squares support vector machine and linear programming support vector machine [18]. In this paper, C-support vector machine is adopted to identify the damage location and extent.

The selection of kernel function is actually the selection of mapping space, and there are many sorts of kernel functions, for example, polynomial kernel function, Gauss radial basis kernel function, Sigmoid kernel function, etc. and the expression for the common polynomial kernel function is shown as follows:

$$
K\left(\boldsymbol{x}_{i}, \boldsymbol{x}_{j}\right)=\left[\left(\boldsymbol{x}_{i} \cdot \boldsymbol{x}_{j}\right)+e\right]^{d},
$$

where $\boldsymbol{x}_{i} \cdot \boldsymbol{x}_{j}$ is the inner product of $\boldsymbol{x}_{i}$ and $\boldsymbol{x}_{j}, e$ and $d$ are constants, and the determination of the values of $e$ and $d$ should be combined with the professional knowledge for specific railway bridge damage identification problem.

\section{Model test}

For verifying the proposed damage identification method, both of the optimization algorithm and the proposed algorithm are applied to the damage identification of a specific continuous girder model bridge [19].

\subsection{Model design}

In accordance with certain real railway bridge dimensions, the model bridge is designed by the 1:20 scale. As shown in Fig. 2, the span arrangement of this model is $5.0+9.6+5.0 \mathrm{~m}$, and the main girder is prestressed concrete single-cell box girder. As shown in Fig. 3, the girder heights are $0.64 \mathrm{~m}, 0.32 \mathrm{~m}$ and $0.32 \mathrm{~m}$ at its intermediate supports section, mid-span section and end section, respectively, and the girder heights are changed as the quadratic parabola function.

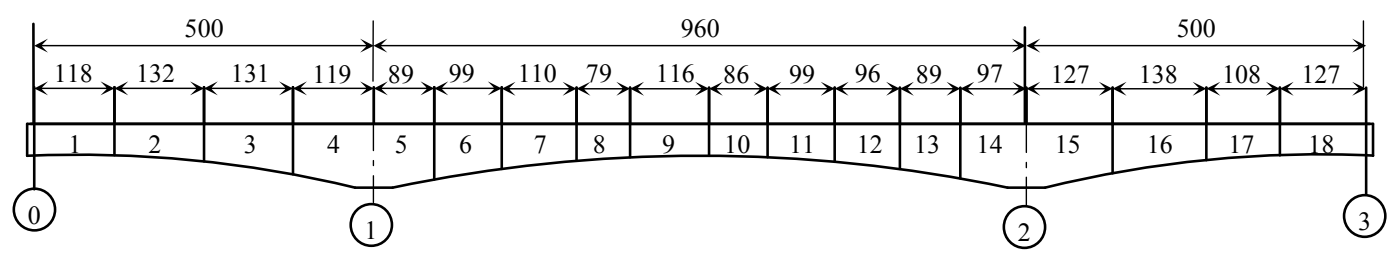

Fig. 2 Elevation of model bridge (unit: $\mathrm{cm}$ )

The theoretical analysis of the model bridge is proceeded firstly, and the first vertical natural frequency is $14.49 \mathrm{~Hz}$, which is greater than the general 3-span continuous girder bridge for the case of no added masses on the model bridge. For obtaining the basis of loading information, the cracking loads and failure loads of different sections under different loading cases are solved by the static analysis, and then the loading procedure is figured out. The fundamental data of damage identification, such as elastic modulus and strength of concrete, are obtained by the material testing as well.

There are 15 deflection measurement points on the whole model bridge, and the whole model bridge is divided into 18 elements in accordance with these deflection measurement points. As shown in Fig. 2, except for four supporting points, the other nodes are all the deflection measurement points. Fig. 4 shows model test site photos. The damage test was implemented at the Structural Laboratory of Southwest Jiaotong University.

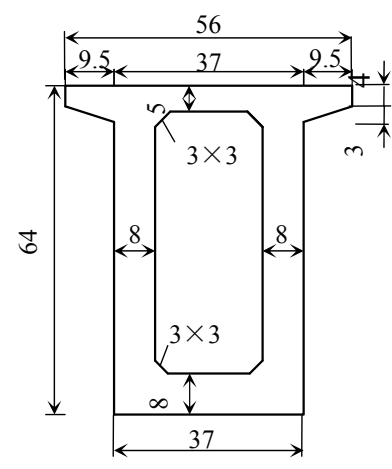

(a) Intermediate supports section

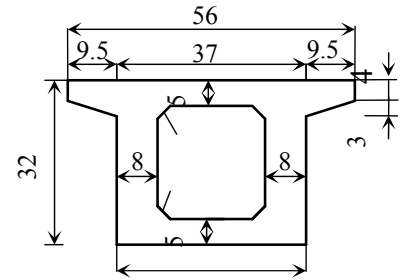

(b) Mid-span section
Fig. 3 Section dimension of model bridge (unit: $\mathrm{cm}$ ) 


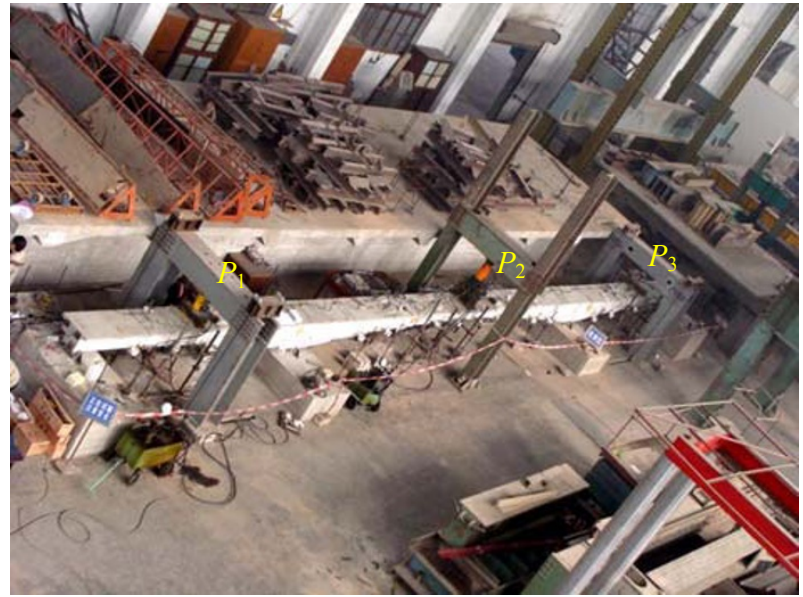

Fig. 4 Damage test of railway continuous girder bridge model

\subsection{Loading procedure}

As shown in Fig. 4, there are three loading point $P_{1}$, $P_{2}$ and $P_{3}$ on the model bridge with $2.2 \mathrm{~m}, 9.8 \mathrm{~m}$ and $17.4 \mathrm{~m}$ distances from the left-side bearing centerline. Two loading cases are introduced in test; all of three loading points are loaded in loading case I and only the two ends loading points are loaded in loading case II.

To verify the functionality of the data acquisition system, pre-load is implemented first, and its loading magnitude is less than the structural cracking load. The formal loading conditions for cases I and II are designed to make the sections located at the middle-span of the main span and the side span crack, respectively. Step-load is introduced to crack the designated section till the peak value is attained, 10 minutes of sustained load at each loading-step, then step-unload. This load procedure is repeated 5 times for keeping cracks.

\subsection{Damage identification}

Assuming the stiffness of intact $i$ th element is $E I_{i}$, and its damaged stiffness is $E I_{i}\left(1-\beta_{i}\right)$, where $\beta_{i}$ is the damage extent of $i$ th element. To obtain the true responses of the model bridge under different loading cases, the integrity and purification of test data are implemented firstly aided by the multivariate statistical analysis [20]. Whereafter, the step-by-step damage identification is carried out as follows.

\subsubsection{Damage early warning}

As mentioned earlier, the process of damage early warning in Section 2.2.1 and the damage identification implementation in Section 2.2, the structural deflection is adopted as the damage early warning index vector, and the binary-classification support vector machine is established with the aid of theoretical damage occur- rence index vectors of the model bridge under variant loading cases and loading magnitudes. The index vector is then built up by the measured data from the experiment [21]; if the discrepancy between theoretical index vector and experimental index vector is less than $5 \%$, the decision of intact structural state is made [19], and the process of damage identification is terminated. And if the discrepancy between theoretical index vector and experimental index vector is greater than $5 \%$, the decision of damaged structural state is made, and then the damage location and extent should be identified. An optimization algorithm with inverse solution and the proposed damage identification method are adopted respectively to detect the structural state after the structure cracks.

In the optimal damage identification process, the optimal variable is the damage extents for each element $\beta_{i}$ $(i=1,2, \cdots, 18)$, the optimal object is the minimal discrepancy between the theoretical deflection and the measured deflection, multiplier method of optimization algorithm is adopted, and the identification result is the numerical solution of damage extent $\beta_{i}$ for each element.

The measured deflections of each measurement point are taken as the damage indexes in the proposed damage identification method in this paper. After normalization of these indexes by Eq. (1), the damage sensitivity analysis is implemented as well to form the rational sample library. The support vector classification and regression machines are adopted to identify the structural damage location and extent, respectively.

\subsubsection{Damage location}

As mentioned earlier, the process of damage location identification in Section 2.1.2. and the damage identification implementation in Section 2.2, C-support vector classification algorithm and kernel function as shown in Eq. (5) are adopted in the damage location identification. In Eq. (5), let $e=0$ and $d=4$, and the kernel function can be written as $K\left(\boldsymbol{x}_{i}, \boldsymbol{x}_{j}\right)=\left(\boldsymbol{x}_{i} \cdot \boldsymbol{x}_{j}\right)^{4}$. Two distinct classifiers are established respectively for loading cases I and II. There are 15 damage cases or 15 classes in each classifier, where single element damage case and multielements damage cases are included, as shown in Table 1. The theoretical responses for the same load applied location under variant load magnitudes are calculated, and then 50 datasets selected from these theoretical responses are considered as the training samples; namely there are 50 samples in each class or damage case. These samples are trained by the support vector classification algorithm and the trained classifier is used to identify the measured deflections. The identification results are shown in Table 2. The identification results by optimization method and experimental results are 
Table 1 Damage cases

\begin{tabular}{cc}
\hline Damage scenario & Damaged element \\
\hline & 3 \\
& 7 \\
& 8 \\
Single element & 9 \\
damage case & 10 \\
& 11 \\
& 12 \\
& 16 \\
& 17 \\
\hline Multi-elements & 2,3 \\
damage case & 9,10 \\
& 16,17 \\
& $2,3,16,17$ \\
& $2,3,9,10,16,17$ \\
\hline
\end{tabular}

also listed in Table 2 for the sake of comparison. As for the identification results of optimization method are the damaged extent $\beta_{i}(i=1,2, \cdots, 18)$ of each element, then the damage location is confirmed according to the value of $\beta_{i}$. The elements with a damage extent greater than 0.1 are listed in Table 2.

In Table 2, the damage location is shown in the form of elements. For example, 1-9 means all elements from 1 to 9 are damaged; the load means the magnitude of applied load; for loading case I, the first value is the load magnitude applied on the mid-span of main span, and the second value is the load magnitude applied both on the mid-spans of two side-spans; for loading case II, the value is the load magnitude applied both on the midspans of two side-spans.

As shown in Table 2, although some identification results obtained by the optimization method are consistent with the cracked location in experiment, such as the identification results of 70-22 $\mathrm{kN}$ and 65-20 $\mathrm{kN}$ load in loading case $\mathrm{I}$, the intact elements in experiment are also misjudged as damaged elements in all of the identification results. Therefore, all results of the conventional optimized damage identification method are unreasonable. Whereas, aided by the proposed damage identification method, the 9th and 10th elements are identified accurately in loading case I; and in loading case II, only the 2 nd and 3rd elements are identified as damaged, but the 16th and 17th elements are not identified as damaged when the load size is $50 \mathrm{kN}$ and $40 \mathrm{kN}$, respectively. The identification results are not consistent with the experimental results in these two applied load magnitudes. Nevertheless, the damaged 2nd, 3rd, 16th and 17 th elements are made out rightly in the other two groups of applied load magnitudes.

Table 2 Comparison of damage location identification results and experimental results

\begin{tabular}{ccccc}
\hline \multirow{2}{*}{ Loading case } & Load $(\mathrm{kN})$ & \multicolumn{2}{c}{ Damaged element } & Experimental cracked element \\
\cline { 3 - 4 } & & Optimization method & Proposed method & \\
\hline I & $70-22$ & $1-11,13-16$ & 9,10 & 9,10 \\
I & $65-20$ & $1-11,14-16$ & 9,10 & 9,10 \\
I & $55-18$ & $1-5,7-9,13-16$ & 9,10 & 9,10 \\
I & $50-15$ & $1-9,11,13-16$ & 9,10 & 9,10 \\
I & $45-15$ & $1-9,11-16$ & 9,10 & 9,10 \\
I & $40-15$ & $1-9,11,13-16$ & 9,10 & 9,10 \\
II & 55 & $2-16$ & $2,3,16,17$ & $2,3,16,17$ \\
II & 50 & $2-10,12-15,17$ & 2,3 & $2,3,16,17$ \\
II & 40 & $4-8,11-16$ & 2,3 & $2,3,16,17$ \\
II & 20 & $1-8,13,14$ & $2,3,16,17$ & $2,3,16,17$ \\
\hline
\end{tabular}

\subsubsection{Damage extent}

Considering the process of damage extent identification in Section 2.1.3 and the damage identification implementation in Section 2.2, variant support vector re- gression machines are set up for each damage location in the damage extent identification. As far as the above mentioned 15 damage locations are concerned, 15 different support vector regression machines are built up. And for each regression machine, $\varepsilon$-support vector re- 
gression algorithm and kernel function shown as Eq. (5) are adopted in the damage extent identification. In Eq. (5), let $e=0$ and $d=1$, and the kernel function can be written as $K\left(\boldsymbol{x}_{i}, \boldsymbol{x}_{j}\right)=\left(\boldsymbol{x}_{i} \cdot \boldsymbol{x}_{j}\right)$. The damage extent is regarded as the identified variant directly, and the same damage extents are taken into consideration especially with regard to the multi-elements damage cases. In the formation of the sample library, the theoretical structural deflections with different damage extents at the same load applied point under variant load magnitudes are calculated, and then 150 datasets selected from these theoretical responses are considered as the training samples. These samples are trained by support vector regression algorithm, and the trained regression machines are introduced to identify the measured deflections. The identification results are shown in Table 3. The damage extent identification will not be implemented if the damage location identification results are not right. Thus, only the two groups with correct damage location are analyzed towards loading case II.

The cracked elements and the maximum crack widths in the experiment and identification results by optimization method are also listed in Table 3 for comparison. The listed damage extents in the optimization method are only the damage extent for the cracked element in the experiment.

As shown in Table 3, multiple cracks are aroused in the structure under the load, and the maximum crack width increases in line with the load magnitude increase, but the difference of maximal crack width between the adjacent elements is small. This shows that the damage extent increases gradually with the increase of load, and the difference of damage extents for neighboring elements is small. The results of the proposed damage extent identification method are in line with the law. Whereas, the damage extent identified by the optimization method hardly changes with the load magnitude and very large difference of damage extents between adjacent elements is obtained. For example, when the load magnitude is $55-18 \mathrm{kN}$ in loading case I, the damage extent for the 9th element is 0.864 , but nearly intact for the 10 th element is only 0.005 . Therefore, the identification

Table 3 Comparison of damage extent identification results and experimental results

\begin{tabular}{|c|c|c|c|c|c|}
\hline \multirow{2}{*}{$\begin{array}{l}\text { Loading } \\
\text { case }\end{array}$} & \multirow{2}{*}{$\begin{array}{l}\text { Load } \\
(\mathrm{kN})\end{array}$} & \multirow{2}{*}{$\begin{array}{l}\text { Cracked } \\
\text { element }\end{array}$} & \multirow{2}{*}{$\begin{array}{l}\text { Maximum crack } \\
\text { width }(\mathrm{mm})\end{array}$} & \multicolumn{2}{|c|}{ Damage extent } \\
\hline & & & & Proposed method & Optimization method \\
\hline \multirow{2}{*}{ I } & \multirow{2}{*}{$70-22$} & 9 & 0.12 & 0.571 & 0.872 \\
\hline & & 10 & 0.11 & 0.571 & 0.114 \\
\hline \multirow{2}{*}{ I } & \multirow{2}{*}{$65-20$} & 9 & 0.10 & 0.514 & 0.874 \\
\hline & & 10 & 0.10 & 0.514 & 0.473 \\
\hline \multirow{2}{*}{ I } & \multirow{2}{*}{$55-18$} & 9 & 0.08 & 0.471 & 0.864 \\
\hline & & 10 & 0.08 & 0.471 & 0.005 \\
\hline \multirow{2}{*}{ I } & \multirow{2}{*}{$50-15$} & 9 & 0.08 & 0.461 & 0.854 \\
\hline & & 10 & 0.07 & 0.461 & 0.056 \\
\hline \multirow{2}{*}{ I } & \multirow{2}{*}{$45-15$} & 9 & 0.07 & 0.459 & 0.833 \\
\hline & & 10 & 0.06 & 0.459 & 0.074 \\
\hline \multirow{2}{*}{ I } & \multirow{2}{*}{$40-15$} & 9 & 0.06 & 0.444 & 0.839 \\
\hline & & 10 & 0.06 & 0.444 & 0.071 \\
\hline \multirow{4}{*}{ II } & \multirow{4}{*}{55} & 2 & 0.16 & 0.687 & 0.438 \\
\hline & & 3 & 0.14 & 0.687 & 0.537 \\
\hline & & 16 & 0.13 & 0.687 & 0.104 \\
\hline & & 17 & 0.12 & 0.687 & 0.050 \\
\hline \multirow{4}{*}{ II } & \multirow{4}{*}{20} & 2 & 0.05 & 0.467 & 0.585 \\
\hline & & 3 & 0.04 & 0.467 & 0.185 \\
\hline & & 16 & 0.05 & 0.467 & 0.013 \\
\hline & & 17 & 0.06 & 0.467 & 0.001 \\
\hline
\end{tabular}


results of optimization method are not matched with the actual results from the experiment.

\subsection{Discussion of damage identification algorithm}

As seen in Table 3, the results of the optimized damage identification method do not match the actual damage cases in experiment, but the results based on the proposed damage identification method are reasonable. The reasons causing the different identification results can be summarized as follows:

(1) Noise immunity ability is different between these two damage identification methods. The optimized damage identification method is sensitive to the test error; it means that small measured data error, such as test error of the applied load value, and measured displacement and elastic modulus, will cause the larger change in the results, and make results unstable. The proposed damage identification method is grounded on the maximum classification surface, so compared with the optimized damage identification method, it has better generalization and stronger noise immunity ability.

(2) The solving method is different between these two damage identification methods. The optimization algorithm is adopted to recognize the damage location and extent in the optimized damage identification method, and the solution can be continuously changed in its feasible solution domain in order to enumerate all possible damages, but it is too easy to fall into the local minimum and makes the result unreliable. The proposed damage identification method based on support vector machine can switch the damage identification problem to a quadratic optimization problem, and the solution is limited to the predefined values in sample library, or the interpolation and extrapolation of predefined values in sample library, so it is easy to obtain the global optimum.

(3) The solution sequence is different for these two damage identification methods. The damage location and extent are identified simultaneously in the optimized damage identification method, and the couple of its optimization algorithm and finite element analysis greatly increases the difficulty of solving the problem. While, the damage location and extent are identified step by step in the proposed damage identification method based on support vector machine; the finite element analysis is carried out at first, and then support vector machines is used to identify the damage location and extent separately. The decoupling of its optimization algorithm and finite element analysis greatly reduces the difficulty of solving the problem.

\section{Conclusions}

With the above analysis and discussion, the following conclusions can be drawn:

(1) The railway bridge damage identification problem is divided into three problems by the proposed step by step damage identification method: damage early warning, damage location and extent identification.

(2) The decoupling of optimization algorithm and finite element analysis reduces the difficulty of damage identification.

(3) The proposed damage identification method based on support vector machine switches the damage identification problem to a quadratic optimization problem, and it is easy to obtain the global optimum. And the application of it to practical railway bridge projects makes it easy to obtain the actual damage state.

(4) The verification results by the model test data show that the proposed damage identification method has better generalization and stronger noise immunity ability than the optimization method. Therefore, the proposed damage identification method can be applied to the damage identification in real railway bridge engineering.

\section{Acknowledgments}

The research is supported by the National Science Foundation (No. 51078316), the Chinese Railway Ministry Scientific Research and Development Program (No. 2011G026-E), and the Sichuan Science and Technology Program (No. 2011JY0032).

\section{References}

[1] C.R. Farrar, N.A.J. Lieven, Damage prognosis: the future of structural health monitoring, http://institute.lanl. gov/ei/shm/pubs/PTRS\%20Prognosis\%2006.pdf, 2006$12-12$.

[2] L.Z. Zhang, Q. Huang, Y.F. Zheng, et al., Research state and advances on bridge structure damage identification theory, Journal of Harbin Institute of Technology, 2005, 37(10): 140-144 (in Chinese).

[3] D.S. Shan, Q. li, C.Y. Fu, et al., Smart Bridge Health Monitoring and Damage Diagnosis, Beijing: China Communication, 2010 (in Chinese).

[4] H. Sohn, C.R. Farrar, F.M. Hemez, et al., A review of structural health monitoring literature: 1996-2001, Los Alamos National Laboratory Report, 2004, LA-13976MS.

[5] J.W. Zhan, H. Xia, S.Y. Chen, et al., Structural damage identification for railway bridges based on train-induced bridge responses and sensitivity analysis, Journal of Sound and Vibration, 2011, 330(4): 757-770.

[6] M.R. Banan, K.D. Hjelmstad, Parameter estimation of structures from static response I: computational aspects, Journal of Structural Engineering, 1994, 120(11): 32433258.

[7] M.R. Banan, K.D. Hjelmstad, Parameter estimation of 
structures from static response II: numerical simulation studies, Journal of Structural Engineering, 1994, 120(11): 3259-3283.

[8] F. Cui, D.Q. Yang, Y. Gao, Bridge structural damage assessment by parameter identification, China Railway Science, 2004, 24(3): 36-39 (in Chinese).

[9] T.Y. Xiang, R.D. Zhao, H.B. Liu, Damage detection prestressed concrete continuous beam from static response, China Civil Engineering Journal, 2003, 36(11): 79-82 (in Chinese).

[10] Q.H. Zhang, Q. Li, L. Tang, Statistical damage assessment method of cable-stayed bridges based on static response test data, Journal of the China Railway Society, 2005, 27(3): 70-75 (in Chinese).

[11] D.S. Shan, C.Y. Fu, Q. Li, Statistical pattern recognition for railway bridge structural damage detection, Bridge Construction, 2011, 205(1): 18-21 (in Chinese).

[12] A.R. Webb, Statistical Pattern Recognition, 2nd ed., New York: John Wiley \& Sons, 2002.

[13] Z.Q. Bian, X.G. Zhang, Pattern Recognition, 2nd ed., Beijing: Tsinghua University Press, 2000 (in Chinese).

[14] W. Zou, Vulnerability Study of Long-Span Continuous Rigid Frame Bridge [Dissertation], Chengdu: Southwest
Jiaotong University, 2009 (in Chinese).

[15] W.H. Jiang, Research on Sample Selection and Its Applications in Pattern Recognition [Dissertation], Nanjing: Nanjing University of Science and Technology, 2007 (in Chinese)

[16] V.N. Vapnik, Statistical Learning Theory, New York: John Wiley and Sons, 1998.

[17] N.Y. Deng, Y.J. Tian, New Method in Data Mining support vector machine, Beijing: Science Press, 2006 (in Chinese).

[18] R.M. Fang, Theory and Application Analysis of Support Vector Machine, Beijing: China Electric Power Press, 2007 (in Chinese).

[19] Southwest Jiaotong University, Intelligent bridge structure, final report (damage identification volumes), Chengdu: Southwest Jiao tong University, 2009 (in Chinese).

[20] J.P. Zhu, Applied Multivariate Statistical Analysis, Beijing: Science Press, 2006 (in Chinese).

[21] Southwest Jiaotong University, Intelligent bridge structure, final report (model test volumes), Chengdu: Southwest Jiaotong University, 2009.

(Editor: Dongju CHEN) 